\title{
Estimação da Energia de Campos Eletromagnéticos em Edificações pela Radiodifusão proveniente de redes IEEE 802.11
}

\author{
Lorenzo Coiado, Alexandre Mota e Lia Mota
}

\begin{abstract}
Lately, a wide variety of devices has been developed which have allowed users access wireless communication net such as smartphones as well as laptops. Due to this a rapid expansion of Wi-Fi IEEE 802.11 standard technology has been felt. Wi-Fi has permitted a greater mobility to users to obtain information and it has become an attractive solution to the corporative world since with no wired net points the cost of installations have been cut down. As Wi-Fi technology is spread, a significant increase of radiated electromagnetic fields has been observed in different installed points. This document presents a method of indoor building electromagnetic measuring areas focused on a 2,4 GHz Wi-Fi net from the potential mapping generated in $\mathrm{dBm}$ by this net. This study has the concern about all harm these electromagnetic radiations may cause to the human being.
\end{abstract}

Index Terms - Radiodifusão, IEEE 802.11, Campos eletromagnéticos

\section{INTRODUÇÃO}

$\mathrm{O}$ ritmo de crescimento da população está cada vez mais acelerado e as cidades passaram a acomodar grande parte da população mundial, criando centros urbanos com alta concentração demográfica. Esse aumento da concentração demográfica tornou-se um grande problema para a gestão das cidades e para as políticas locais. Existe, atualmente, uma grande pressão para a otimização de recursos e para tornar as cidades mais eficientes. Iniciativas por parte do setor privado e governo passaram a investir em tecnologia de informação e comunicação (TIC) para tentar minimizar os problemas de infraestrutura urbana. Nos dias atuais, a Internet prevê a conexão de sensores a serem utilizados como um recurso eficiente em Cidades Inteligentes [1].

A disseminação da informação veio acompanhada de uma tecnologia de rede que se expandiu também em grande proporção: o padrão IEEE 802.11 (Wi-Fi). Esse padrão

Lorenzo Coiado, Alexandre Mota e Lia Mota atuam junto ao Centro de Ciências Exatas, Ambientais e de Tecnologias da Pintifícia Universidade Católica de Campinas, Rod. Dom Pedro I, Km 136, CEP 13086-900, Brasil (e-mails: lorenzo.coiado@gmail.com, amota@puc-campinas.edu.br, lia.mota@puc-campinas.edu.br). proporciona grande mobilidade e conectividade a uma vasta quantidade de dispositivos, tais como: tablets, televisores, palmtops, impressoras, câmeras de segurança. A tecnologia Wi-Fi veio otimizar recursos e facilitar a interatividade dos usuários com a rede [2]. Por ser uma tecnologia sem fio permite uma grande redução de custos em projetos, uma vez que elimina a necessidade de pontos cabeados, e permite que infraestruturas prediais obsoletas possam ter uma solução de acesso à Internet sem a necessidade de transpor cabos.

Assim, um agente invisível e que pode causar danos à saúde humana está cada vez mais presente em nossas vidas: o campo eletromagnético em edificações pela radiodifusão proveniente de redes IEEE 802.11. Todas as redes sem fio Wi-Fi lançam radiações eletromagnéticas no ambiente de sua atuação. E com a disseminação desta tecnologia, houve um aumento significativo de pontos de acessos nas edificações. Nesse contexto, este trabalho tem como objetivo desenvolver uma metodologia de medição desses campos irradiados, através do levantamento da potência $(\mathrm{em} \mathrm{dBm})$ gerada por uma rede IEEE 802.11 , com a preocupação de observar a energia absorvida pelo corpo humano ao ser exposto a este agente. A Figura 1 ilustra a termografia de um corpo humano [3].

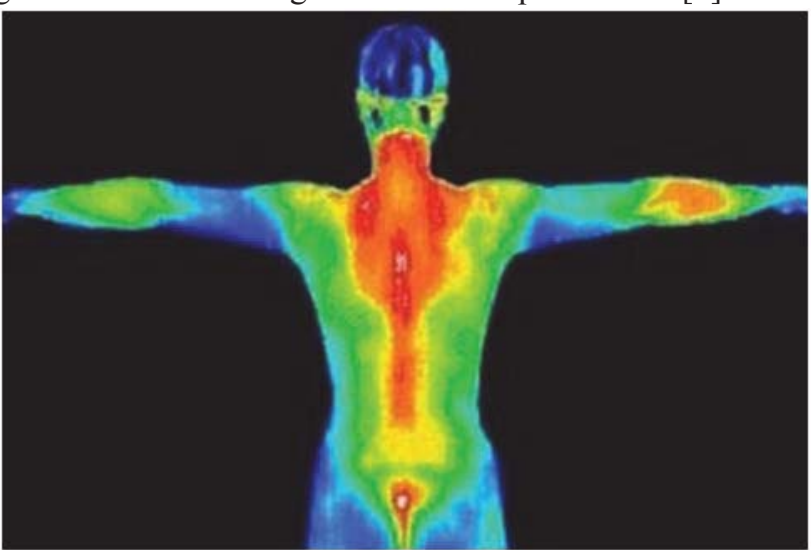

Fig. 1. Termografia de um corpo humano. Adaptado de [3].

\section{CAmpos EletromagnÉticos}

\section{A. Equações de Maxwell}

A base teórica para estudo de campos eletromagnéticos corresponde às equações publicadas, em 1864, pelo físico escocês James Clerk Maxwell, que em seu trabalho intitulado "Dynamical Theory of Eletromagnetic Field" unificou as 
equações de campo elétrico e campo magnético. Segue uma breve descrição de cada uma delas [4].

$\oint E \cdot d A=\frac{q}{\varepsilon_{0}}$

$\oint B . d A=0$

$\oint E . d S=-\frac{d \Phi B}{d t}$

$\oint B \cdot d S=\mu_{0} I+\varepsilon_{0} \mu_{0} \frac{d \Phi E}{d t}$

Onde "E" é o campo elétrico, "B" é a densidade de fluxo magnético, "q" é a carga elétrica, " $\varepsilon_{0}$ " é a permissividade do vácuo, " $\mu_{0}$ " é a permeabilidade do vácuo, "I" é a corrente elétrica, “ $\phi B$ " é o fluxo magnético e " $\phi E$ " é o fluxo elétrico.

A Equação (1) é a Lei de Gauss e afirma que, para uma superfície fechada, o campo elétrico é proporcional à carga resultante contida no volume fechado pela superfície.

A Equação (2) afirma que, o fluxo do campo magnético para uma superfície fechada é zero, não existindo o análogo magnético para a carga elétrica.

A Equação (3) é a Lei de Faraday, e afirma que a integral de linha do campo elétrico ao longo de um trajeto fechado é proporcional à taxa de variação em relação ao tempo do fluxo magnético para uma superfície delimitada por este trajeto.

A Equação (4) é uma forma alterada da Lei de Ampère e afirma que a integral de linha do campo magnético ao longo de um trajeto fechado é proporcional à soma de dois termos. $\mathrm{O}$ primeiro contém a corrente resultante que flui através de uma superfície delimitada pelo trajeto fechado. E o segundo contem a taxa de variação, no tempo, do fluxo do campo elétrico para uma superfície delimitada pelo trajeto.

Assim, as equações de Maxwell fazem uma correlação entre os campos elétricos e magnéticos [4].

\section{B. Energia e Potência}

Os campos eletromagnéticos (medidos em $\mathrm{V} / \mathrm{m}$ ) têm a capacidade de transportar energia, sendo que a potência (P) corresponde à energia desenvolvida ou dissipada em um certo intervalo de tempo. Essas grandezas são fundamentais para que as redes sem fio funcionem, uma vez que os receptores necessitam de uma potência mínima para reconhecimento do sinal [5].

A potência contida no campo elétrico é proporcional ao quadrado da intensidade do campo elétrico, conforme Equação (5).

$$
21^{\prime 6}
$$

\section{Metodologia}

\section{A. Hardware}

Para os testes realizados neste trabalho, foi utilizado um computador desktop, com sistema operacional Linux Ubuntu e placa de rede configurada como Access Point (AP) numa rede Wi-Fi de 2.4GHz (padrão IEEE 802.11). Para o acesso remoto ao computador, foi utilizado um notebook DELL Inspiration, Intel Core i5. A bancada de testes está ilustrada na Figura 2.

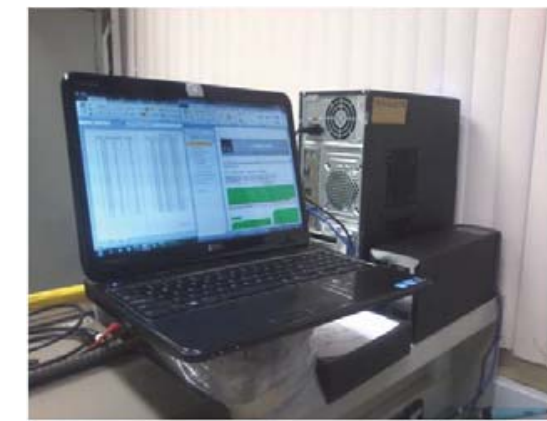

Fig. 2. Bancada de testes.

\section{B. Software}

No computador torre foi utilizado o Linux Ubuntu, por ser um sistema operacional que permite acesso ao hardware da placa de rede e com maiores funções do que um sistema operacional comercial. Foram utilizados scripts de visualização da potência recebida no AP em $\mathrm{dBm}$. Para o acesso remoto ao computador, foi utilizado um notebook com sistema operacional Windows 7 e software PuTTY, que permite o acesso remoto ao Access Point.

\section{Script de acesso}

O script permite a visualização da potência recebida em $\mathrm{dBm}$ por um $\mathrm{AP}$, de um laptop conectado na rede Wi-Fi desta base. Com o afastamento ou aproximação do laptop ao AP, é possível verificar a variação da potência do sinal recebido. $\mathrm{O}$ script foi definido para coletar dados a cada um segundo.

\section{Access Point}

Em um link padrão IEEE 802.11, a potência é medida em miliwatts ou dBm. As medidas tomadas com o AP têm como saída a potência em $\mathrm{dBm}$. Para transformá-la em energia, são utilizadas as seguintes equações.

$$
\begin{aligned}
& / \mathrm{L} \operatorname{srHK} \frac{\mathrm{C}}{5-} \\
& \check{\operatorname{sr}}^{\mathrm{W}_{54}} \text { sI9 L } 2
\end{aligned}
$$

As equações (6) e (7) têm como objetivo a transformação da potencia em dBm, fornecida pelo Access Point, para miliWatt. Sendo que o "M" é a medida da potência recebida no AP, coletada no experimento e "P" é a potência em miliWatt. Sabendo-se que 1 Watt é igual a 1 Joule/segundo, é possível determinar a Energia irradiada por determinado período de tempo.

\section{COLETA DE DAdOS}

\section{A. Primeiro experimento}

Em um primeiro momento, foi feita a medição da potência recebida no AP durante 5 minutos, com intervalo de 1 segundo entre as medidas. A Figura 3 ilustra o esquema utilizado para o primeiro experimento (sem obstáculos entre laptop e AP). Neste primeiro experimento, a energia irradiada (E) pelo AP foi de $7,88 \times 10^{-7}$ Joules. 


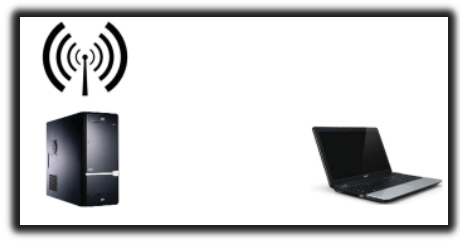

Fig. 3. Medição sem obstáculos (primeiro experimento).

\section{B. Segundo experimento}

No segundo experimento, foi feita a medição da potência do AP durante o mesmo período de tempo, com uma pessoa servindo de obstáculo. A Figura 4 ilustra o esquema utilizado para o segundo experimento (com uma pessoa funcionando como obstáculo entre laptop e AP). Neste segundo experimento, a energia irradiada (E) pelo AP foi de 5,74 $\times 10^{-8}$ Joules.

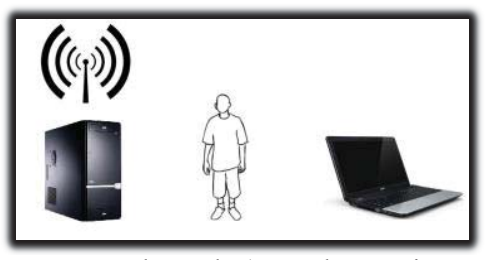

Fig. 4. Uma pessoa como obstáculo (segundo experimento).

\section{Terceiro experimento}

No terceiro experimento, foi feita a medição da potência do AP durante o mesmo período de tempo, com um muro como obstáculo. A Figura 5 ilustra o esquema utilizado para o terceiro experimento (com um muro como obstáculo entre laptop e AP). Neste terceiro experimento, a energia irradiada (E) pelo AP foi de $2,98 \times 10^{-9}$ Joules.

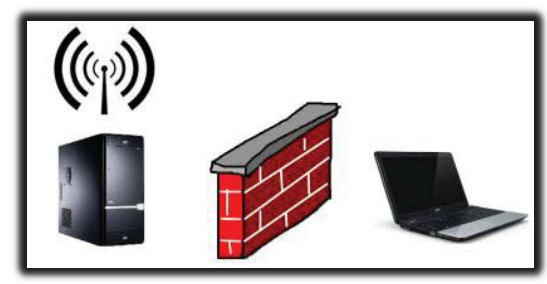

Fig. 5. Um muro como obstáculo (terceiro experimento).

\section{Resultados E Discussão}

A colocação de barreiras (corpo humano no segundo experimento e muro no terceiro experimento) entre o AP e o notebook teve como objetivo estimar a quantidade de energia absorvida por esses obstáculos, comparando os resultados do segundo e do terceiro experimentos com o caso base sem nenhum obstáculo (primeiro experimento).

Os resultados dos três experimentos podem ser sintetizados de acordo com a Tabela I.

Tabela I: Energia irradiada para os três experimentos

\begin{tabular}{|l|l|}
\hline Experimento & Energia Irradiada $(\mathrm{J})$ \\
\hline Primeiro & $\mathrm{E}_{1}=7,88 \times 10^{-7}$ \\
\hline Segundo & $\mathrm{E}_{2}=5,74 \times 10^{-8}$ \\
\hline Terceiro & $\mathrm{E}_{3}=2,98 \times 10^{-9}$ \\
\hline
\end{tabular}

Mais especificamente, a partir dos resultados coletados, pode-se estimar a quantidade de energia absorvida pelo corpo humano $\left(\mathrm{E}_{\mathrm{CH}}\right)$ ao ser exposto a uma rede Wi-Fi durante 5 minutos, de acordo com a Equação (8).

$\mathrm{E}_{\mathrm{CH}}=\mathrm{E}_{1}-\mathrm{E}_{2}=\left(7,88 \times 10^{-7}\right)-\left(5,74 \times 10^{-8}\right)=7,30 \times 10^{-7} J(8)$
A título de comparação, pode-se estimar a quantidade de energia absorvida por um muro $\left(\mathrm{E}_{\mathrm{M}}\right)$ ao ser exposto a uma rede Wi-Fi durante 5 minutos, de acordo com a Equação (9). $\mathrm{E}_{\mathrm{CH}}=\mathrm{E}_{1}-\mathrm{E}_{3}=\left(7,88 \times 10^{-7}\right)-\left(2,98 \times 10^{-9}\right)=7,85 \times 10^{-7} J(9)$

Cabe ressaltar que, neste trabalho, não foi considerada a quantidade de energia que pode ser refletida pelos corpos.

\section{CONCLUSÕES}

Como conclusões deste trabalho, pode-se destacar que foi possível estimar a energia associada aos campos eletromagnéticos em edificações pela radiodifusão proveniente de redes Wi-Fi padrão IEEE 802.11. A partir dos resultados obtidos, pôde-se concluir que o corpo humano absorve energia proveniente das redes Wi-Fi IEEE 802.11 e, mais ainda, pôdese estimar essa energia absorvida. É de se esperar que a quantidade de energia absorvida pelo corpo esteja relacionada com o tempo de exposição do mesmo, com a quantidade de APs no ambiente e com possíveis danos à saúde, aspectos que deverão ser tratados em trabalhos futuros.

\section{AGRADECIMENTO}

Os autores agradecem à Pontifícia Universidade Católica de Campinas (PUC-Campinas) e ao CNPq pelo suporte a este trabalho.

\section{REFERENCES}

[1] P. Charith, et al. "Sensing as a service model for smart cities supported by Internet of Things". Wiley Online Library.

[2] C.P.C. Marques. Identificação de ofensores via análise da sensibilidade de estações na vazão de redes IEEE $802.11,2013$

[3] Termografiainfrarroja, www.investigacionengestiondeportiva.es.

Available: $05 / 2014$

Access:

[4] F. J. Keller, et al. Física. Clemson University. Vol. 2, 1999.

[5] Pietrosemoli."Wireless Networking in the Developing Word.Third Edition", 2013.

\section{Cite this article:}

Coiado, L., Mota, A. e Mota, L.; 2015. Estimação da Energia de Campos Eletromagnéticos em Edificações pela Radiodifusão proveniente de redes IEEE 802.11. SET EXPO PROCEEDINGS. ISSN Print: 2447-0481.ISSN Online: 2447-049X. v.1.doi: 10.18580/setep.2015.1.3 Web-link: http:// dx.doi.org/10.18580/setep.2015.1.3 